\title{
Recurrent Multiplicative Neuron Model Artificial Neural Network for Non-Linear Time Series Forecasting
}

\author{
Erol Egrioglu ${ }^{a}$, Ufuk Yolcu ${ }^{b}$, Cagdas Hakan Aladag $^{c}$, Eren Bas ${ }^{b}$ \\ ${ }^{a}$ Ondokuz Mayls University, Faculty of Arts and Science Department of Statistics, Samsun and 55139, Turkey \\ ${ }^{b}$ Giresun University, Faculty of Arts and Science Department of Statistics, Giresun and 28000, Turley \\ ${ }^{c}$ Hacettepe University, Faculty of Science Department of Statistics, Ankara and 06200, Turkey
}

\begin{abstract}
Artificial neural networks (ANN) have been widely used in recent years to model non-linear time series since ANN approach is a responsive method and does not require some assumptions such as normality or linearity. An important problem with using ANN for time series forecasting is to determine the number of neurons in hidden layer. There have been some approaches in the literature to deal with the problem of determining the number of neurons in hidden layer. A new ANN model was suggested which is called multiplicative neuron model (MNM) in the literature. MNM has only one neuron in hidden layer. Therefore, the problem of determining the number of neurons in hidden layer is automatically solved when MNM is employed. Also, MNM can produce accurate forecasts for non-linear time series. ANN models utilized for non-linear time series have generally autoregressive structures since lagged variables of time series are generally inputs of these models. On the other hand, it is a wellknown fact that better forecasts for real life time series can be obtained from models whose inputs are lagged variables of error. In this study, a new recurrent multiplicative neuron neural network model is firstly proposed. In the proposed method, lagged variables of error are included in the model. Also, the problem of determining the number of neurons in hidden layer is avoided when the proposed method is used. To train the proposed neural network model, particle swarm optimization algorithm was used. To evaluate the performance of the proposed model, it was applied to a real life time series. Then, results produced by the proposed method were compared to those obtained from other methods. It was observed that the proposed method has superior performance to existing methods.
\end{abstract}

(C) 2014 The Authors. Published by Elsevier Ltd. Open access under CC BY-NC-ND license.

Selection and peer review under responsibility of Organizing Committee of BEM 2013.

Keywords: Artificial neural networks, forecasting, multiplicative neuron model, non-linear time series, recurrent neural networks.

\section{Introduction}

Various different methods have been used to forecast non-linear real-world time series in the literature (Aladag and Egrioglu, 2012). These methods can be grouped as probabilistic methods, the methods based on fuzzy set theory and the methods based on artificial neural network. In recent years, there have been many studies which focus on ANN. Different approaches can be utilized when time series are forecasted with ANN. In these approaches, lagged variables of time series or more than one time series can be used as input values. The first one has been usually preferred in the literature. Multilayer perceptron neural networks are extensively used to forecast time series. The

\footnotetext{
* Corresponding Author: Erol Egrioglu. Tel.: +090-362-312-1919

E-mail address: erole@omu.edu.tr
} 
literature related to usage of this kind of neural network for time series forecasting was reviewed by Zhang et al. (1998) and Zhang (2003). Multilayer perceptron uses McCuloch\&Pitts neuron model which is based on an additive aggregation function. Thus, the output of the multilayer perceptron can be considered as non-linear transformation of sum of the inputs. Activation function provides non-linearity in here. In a multilayer perceptron which includes more than one neuron in the hidden layer, the output is a non-linear function of multiplication of weighted sum of the inputs. Especially, the number of neurons in hidden layer directly affects the performance of multilayer perceptron neural networks. Therefore, determination of the number of neurons in hidden layer is a vital issue. Egrioglu et al. (2008), Aladag et al. (2010), Aladag (2011) proposed some methods to determine the number of neurons in hidden layer and inputs of the model.

Yadav et al. (2007) introduced multiplicative neuron model ANN (MNM-ANN) which has only one neuron in the hidden layer. This kind of neural network is different from multilayer perceptron neural network in aspect of the neuron model included. MNM-ANN is composed of multiplicative neuron model instead of McCuloch\&Pitts neuron model. In multiplicative neuron model, aggregation function is not additive but multiplicative. Hence, the output of MNM-ANN is a non-linear function of multiplication of the inputs. This multiplicative structure strengthens non-linearity characteristic of the model. MNM-ANN uses less parameter than those employed by multilayer perceptron neural networks since it has only one neuron in the hidden layer. For time series forecasting, different ANN based on multiplicative neuron model such as linear and non-linear ANN (L\&NL-ANN) and multiplicative seasonal artificial neural network (MS-ANN) were proposed by Yolcu et al. (2013) and Aladag et al. (2013), respectively.

In probabilistic models used for time series forecasting, inputs are lagged variables of time series (autoregressive terms) and lagged variables of error (moving average terms). Utilizing moving average (MA) terms in these models is as effective as using autoregressive (AR) terms. On the other hand, when ANN models are used for time series forecasting, AR terms are usually employed as inputs and MA terms are not taken into consideration. In addition to AR terms, if MA terms are also used, more accurate forecasts will be obtained. When MA terms are utilized, it is necessary to make important adjustments in ANN architectures and training algorithms of these architectures. Like using MA terms, Elman neural networks (Elman, 1990) have a mechanism in which neurons in context layer are fed from the hidden layer. However, Elman neural networks do not exactly including a MA term. To incorporate MA terms into ANN, the architecture should have a recurrent feedback structure from output layer. Jordan (1986) suggested a recurrent architecture structure in which neurons in context layer are fed from the output layer. Jordan's recurrent architecture structure is proper only for one step lag. However, it is a well-known fact capability of using more than one step lag cause an increase in forecasting performance of ANN. In some studies available in the literature such as Buhamra et al. (2003), Egrioglu et al. (2009), and Khashei and Bijari (2010), hybrid methods were proposed and lagged variables of error were taken as inputs of ANN. On the other hand, in these studies, lagged variables of error were obtained from Box-Jenkins (1976) models instead of ANN models. In the literature, an artificial neural network model which uses lagged variables of its own error for feedback has not proposed yet. In other words, an artificial neural network model that has ARMA $(p, q)$ structure does not exist in the literature. In this study, a novel artificial neural network model which has ARMA $(p, q)$ structure and based on multiplicative neuron model is proposed for time series forecasting. The proposed model is the first artificial neural network model which has ARMA structure. In the next section, the proposed model is introduced and the algorithm, which is based on particle swarm optimization (PSO), for training of this model is presented. In section 3, the proposed model is applied to a real-world time series. Finally, the last section concludes the paper.

\section{The Proposed Model}

Although MNM-ANN firstly proposed by Yadav et al. (2007) has proved its success on forecasting problems, a major drawback of the method is that MNM-ANN does not have a recurrent feedback mechanism. In the time series literature, it is a well-known fact that using MA terms in forecasting models is as effective as using AR terms. In 
this study, a new recurrent ANN model based on multiplicative neuron model is proposed. The proposed model is called recurrent multiplicative neuron artificial neural network model (RMNM-ANN). In the proposed model, in addition to AR terms, MA terms are also incorporated into the model by feedbacking own error of the model. The architecture of the proposed ANN model is given in Figure 1. In this figure, $L$ and $e_{t}$ are backshift operator and error for time $t$ respectively, so $L e_{t}=e_{t-1} . X_{t}$ represents observation value for time $t . f$ represents activation function which provides non-linear mapping. Sigmoid function is used as activation function in this study since this activation function is widely used in the literature. Output $t_{t}$ and Desired $_{t}$ are the output value and target value of the model for time $t$, respectively.

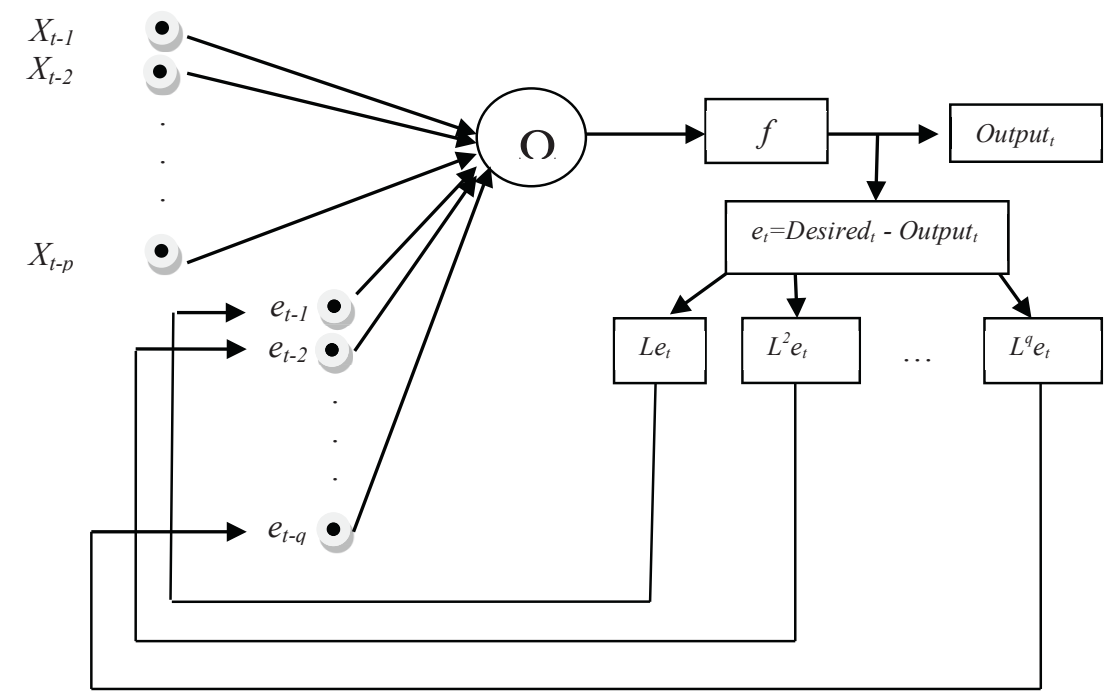

Figure 1. The Architecture of the proposed RMNM-ANN model

The algorithm of the calculation of the output values of the proposed method is given below.

\section{Algorithm 1. Calculation of the output values of the proposed RMNM-ANN model}

Let $n$ be the number of learning samples. First of all, the number of input of RMNM-ANN is determined, that is, values of $p$ and $q$ are decided. Then, according to these values of $p$ and $q$, the outputs of the proposed RMNM-ANN model can be computed as follows:

Step 1. Initialize the loop counter $k(k=0)$.

Step 2. Increase $k$ by $1(k=k+1)$. Calculations for $k^{\text {th }}$ learning sample are performed.

The inputs of RMNM-ANN are $X_{t-1}, X_{t-2}, \ldots, X_{t-p}, e_{t-1}, e_{t-2}, \ldots, e_{t-q}$. As seen from Figure 1, RMNM-ANN has one neuron in the hidden layer. Activation value of the neuron is represented by net and obtained from multiplication of inputs of RMNM-ANN by corresponding weights. When $k=1, e_{t-1}, e_{t-2}, \ldots, e_{t-q}$ are taken as 0 since the output of RMNM-ANN has not been calculated yet. When $k=2, e_{t-1}$ can be calculated. $e_{t-1}$ equals to (Desired ${ }_{t}-$ Output $_{t}$ ) since the output of RMNM-ANN for the first learning sample was obtained. However, $e_{t-2}, \ldots, e_{t-q}$ are taken as 0 . In a similar way, last q $-\mathrm{k}$ terms of $e_{t-1}, e_{t-2}, \ldots, e_{t-q}$ will be taken as 0 for $k \leq q$. If $k>q$ then, each $e_{t-i}(i=1,2, \ldots, q)$ can be calculated. Let $W X_{i}$ and $b X_{i}(i=1,2, \ldots, p)$ be weights which connect $X_{t-1}, X_{t-2}, \ldots, X_{t-p}$ inputs to the neuron and the related bias values, respectively. Let $W E_{i}$ and $b E_{i}(i=1,2, \ldots, q)$ be weights which connect $e_{t-1}, e_{t-2}, \ldots, e_{t-q}$ 
inputs to the neuron and the related bias values, respectively. Thus, for $k^{\text {th }}$ learning sample, activation value of the neuron net $_{k}$ can be calculated using the formula (1).

$n e t_{k}=\prod_{i=1}^{p}\left(W X_{i} \times X_{t-i}+b X_{i}\right) \times \prod_{j=1}^{q}\left(W E_{j} \times e_{t-j}+b E_{j}\right)$

Step 3. Calculate the output value of RMNM-ANN by using net $_{k}$ obtained in the previous step and the activation function $f$ as follows:

Output $_{t}=f\left(\right.$ net $\left._{\mathrm{k}}\right)=\frac{1}{1+\exp \left(-n e t_{k}\right)}$

Step 4. Calculate error $e_{t}$ based on the difference between the obtained output value and desired value by using the formula given below.

$e_{t}=$ Desired $_{t}-$ Output $_{t}$

This $e_{t}$ value will be used as an input of RMNM-ANN for the next learning sample.

Step 5. If $k \leq n$, then go to Step 2. Otherwise, terminate the algorithm.

PSO is utilized in order to train the proposed RMNM-ANN model. PSO introduced by Kenedy ve Eberhart (1995) is an intelligent optimization technique. In many applications, PSO method has produced better results than those produced by other methods such as gradient descent and Newton methods which require derivative. Especially, when it is very hard to calculate derivatives, good results can be obtained using PSO. Therefore, this optimization method has drawn a great amount of attention in recent years. For the architecture structure of the proposed RMNMANN model, it can be very hard to obtain derivatives so PSO is utilized to train the proposed model. In the PSO algorithm, positions of a particle are weights of proposed RMNM-ANN model. Hence, a particle has $2(p+q)$ positions. Structure of a particle is illustrated in Figure 2. The algorithm of the PSO method which is used to train the proposed model is given below.

The autoregressive component's weights and The moving average component's weights and

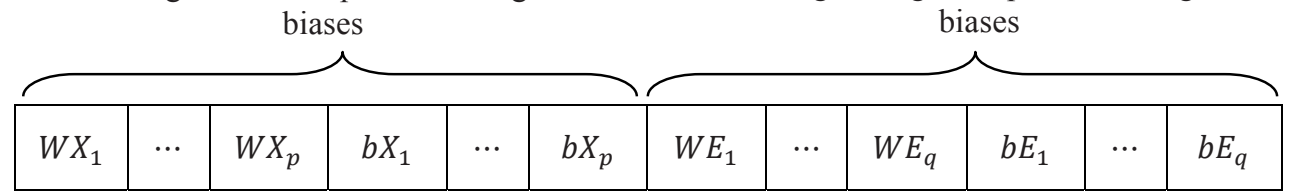

Figure 2. Structure of a particle

Algorihtm 2. PSO algorithm used to train the proposed RMNM-ANN model

Step 1. Positions of each $k^{\text {th }}(\mathrm{k}=1,2, \ldots, p n)$ particles' positions and velocities are randomly determined and kept in vectors $X_{k}$ and $V_{k}$ given as follows:

$X_{k}=\left\{x_{k, 1}, x_{k, 2}, \ldots, x_{k, d}\right\}, k=1,2, \ldots, p n$

$V_{k}=\left\{v_{k, 1}, v_{k, 2}, \ldots, v_{k, d}\right\}, k=1,2, \ldots, p n$

where $x_{i}^{k}(i=1,2, \ldots, d)$ represents $i$ th position of $k$ th particle. $p n$ and $d$ represents the number of particles in a swarm and positions, respectively. The initial positions and velocities of each particle in a swarm are randomly generated from uniform distribution $(0,1)$ and $(-v m, v m)$, respectively. 
Step 2. The parameters of PSO are determined.

In the first step, the parameters which direct the PSO algorithm are determined. These parameters are $p n, v m, c_{1 i}, c_{1 f}$, $c_{2 i}, c_{2 f}, w_{1}$, and $w_{2}$. Let $c_{1}$ and $c_{2}$ represents cognitive and social coefficients, respectively, and $w$ is the inertia parameter. Let $\left(c_{1 i}, c_{1 f}\right),\left(c_{2 i}, c_{2 f}\right)$, and $\left(w_{1}, w_{2}\right)$ be the intervals which includes possible values for $c_{1}, c_{2}$ and $w$, respectively. At each iteration, these parameters are calculated by using the formulas given in (6), (7) and (8).

$c_{1}=\left(c_{1 f}-c_{1 i}\right) \frac{t}{\operatorname{maxt}}+c_{1 i}$

$c_{2}=\left(c_{2 f}-c_{2 i}\right) \frac{t}{\max t}+c_{2 i}$

$w=\left(w_{2}-w_{1}\right) \frac{\max t-t}{\max t}+w_{1}$

Step 3. Evaluation function values are computed. Evaluation function values for each particle are calculated. MSE given in below is used as evaluation function.

$M S E=\frac{1}{n} \sum_{t=1}^{n}\left(\text { desired }_{t}-\text { output }_{t}\right)^{2}$

where $n$ represents the number of learning sample. The output value of the proposed model is calculated by algorithm 1 .

Step 4. Pbest $(k=1,2, \ldots, p n)$ and Gbest are determined due to evaluation function values calculated in the previous step. Pbest $t_{k}$ is a vector stores the positions corresponding to the $k^{\text {th }}$ particle's best individual performance, and Gbest is the best particle, which has the best evaluation function value, found so far.

Pbest $_{k}=\left(p_{k, 1}, p_{k, 2}, \ldots, p_{k, d}\right), k=1,2, \ldots, p n$

Gbest $=\left(p_{g, 1}, p_{g, 2}, \ldots, p_{g, d}\right)$

Step 5. The parameters are updated. The updated values of cognitive coefficient $c_{1}$, social coefficient $c_{2}$, and inertia parameter $w$ are calculated using the formulas given in (6), (7), and (8).

Step 6. New values of positions and velocities are calculated. New values of positions and velocities for each particle are computed by using the formulas given in (12) and (13). If maximum iteration number is reached, the algorithm goes to Step 3; otherwise, it goes to Step 7.

$v_{i, d}^{t+1}=\left[w \times v_{i, d}^{t}+c_{1} \times \operatorname{rand}_{1} \times\left(p_{i, d}-x_{i, d}\right)+c_{2} \times \operatorname{rand}_{2} \times\left(p_{g, d}-x_{i, d}\right)\right]$

$x_{i, d}^{t+1}=x_{i, d}+v_{i, d}^{t+1}$

Step 7. The optimal solution is determined. The elements of Gbest are taken as the optimal weight values of the new ANN model.

\section{The Application}

The real-world time series used in the implementation is the amount of carbon dioxide measured monthly in Ankara capitol of Turkey (ANSO) between March 1995 and April 2006. The graph of time series data of the amount of $\mathrm{SO}_{2}$ in Ankara is given in Figure 3. This time series has both trend and seasonal components and its period is 12 . The first 124 observations are used for training and the last 10 observations are used for test set. In addition to the proposed approach, seasonal autoregressive integrated moving average (SARIMA), Winter's multiplicative 
exponential smoothing (WMES), MLP-ANN, radial bases function ANN (RBF-ANN), E-ANN, MNM-ANN, MSANN and L\&NL-ANN methods are also used to analyze ANSO data. For the test set, mean square error (MSE) and mean absolute percentage error (MAPE) values produced by all methods are summarized in Table 1. MAPE is calculated by formula (14).

MAPE $=\frac{1}{n} \sum_{t=1}^{n}\left|\frac{\text { desired }_{t}-\text { output }_{t}}{\text { desired }_{t}}\right|$

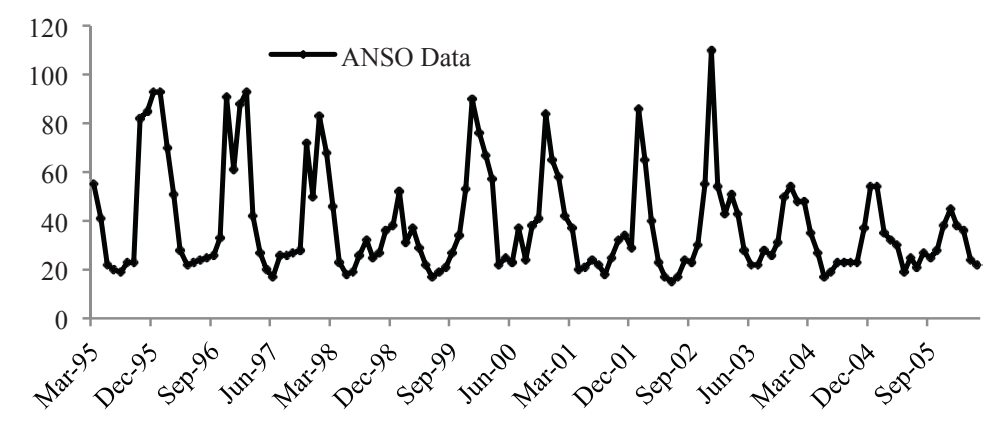

Figure 3. The time series data of the amount of $\mathrm{SO}_{2}$ in Ankara.

In the training process of L\&NL-ANN, MS-ANN and proposed RMNM-ANN model, the parameters of the PSO are determined as follows: $\left(c_{1 i}, c_{1 f}\right)=(2,3),\left(c_{2 i}, c_{2 f}\right)=(2,3),\left(w_{1}, w_{2}\right)=(1,2), p n=30$, and $\operatorname{maxt}=1000$. For the proposed RMNM-ANN model, the best result was obtained when $p=3$ and $q=13$. To determine the best values of $p$ and $q$, trial and error method was employed. According to Table 1, it is clearly seen that the best results in terms of both performance measures were obtained when the proposed RMNM-ANN model was used.

Table 1 The obtained forecasting results for ANSO data

\begin{tabular}{cccccccccc}
\hline Test Data & SARIMA & WMES & $\begin{array}{c}\text { MLP- } \\
\text { ANN }\end{array}$ & RBF-ANN & E-ANN & $\begin{array}{c}\text { MNM- } \\
\text { ANN }\end{array}$ & MS-ANN & $\begin{array}{c}\text { L\&NL- ANN } \\
\text { RMNM- } \\
\text { ANN }\end{array}$ \\
\hline MSE & 92.6387 & 50.4980 & 13.9891 & 106.4797 & 13.4821 & 40.2006 & 9.1010 & 12.7263 & 8.6289 \\
MAPE & 0.2336 & 0.2204 & 0.0995 & 0.3248 & 0.0990 & 0.1822 & 0.0887 & 0.0944 & 0.0761 \\
\hline
\end{tabular}




\section{Conclusion}

Although ANN models for non-linear time series use lagged variables of time series, they do not take lagged variables of error into account. Some hybrid approaches in the literature use lagged variables of error but these lagged variables are obtained from other approaches such as Box-Jenkins models. A new recurrent ANN model based on multiplicative neuron model is suggested in this study. The proposed RMNM-ANN model can produce lagged variables of error and use them as inputs because of the recurrent feedback structure it has. Also, unlike the most of the other ANN models, it does not the problem of determination of the number of neurons in hidden layer. Since it has only one neuron in hidden layer, it can reach results with less parameter. When the proposed RMNMANN model is applied, parameters needed to be determined are the number of lagged variables for time series and error. These parameters were determined using trial and error method in this study. The proposed model was applied to a real-world time series and the obtained results are compared to results produced by other methods available in the literature. It was observed that the proposed model produced the most accurate forecasts. In future studies, to determine the parameters of RMNM-ANN model, different systematic approaches can be utilized instead of trial and error method.

\section{References}

Aladag, C.H. (2011). A new architecture selection method based on tabu search for artificial neural networks, Expert Systems with Applications, $38,3287-3293$

Aladag C.H., Yolcu U., \& Egrioglu E. (2013). A new multiplicative seasonal neural network model based on particle swarm optimization, Neural Processing Letters, DOI 10. 1007/S11063-012-9244-y.

Aladag C.H., Egrioglu E., Editors (2012). Advances in time series forecasting, Bentham Science Publishers Ltd., eISBN: 978-1-60805-373-5. (http://www.benthamscience.com/ebooks/9781608053735/index.htm)

Aladag C.H., Egrioglu E., Gunay S., \& Basaran M.A. (2010). Improving weighted information criterion by using optimization, Journal of Computational and Applied Mathematics, 233, 2683-2687.

Box G.E.P., Jenkins G.M. (1976). Time Series Analysis: Forecasting and Control, San Francisco, Holdan-Day CA, (Chapter 4).

BuHamra S., Smaoui N., Gabr M. (2003). The Box-Jenkins analysis and neural networks: prediction and time series modeling, Applied Mathematical modeling, 27, 805-815.

Egrioglu E., Aladag C.H., \& Gunay S. (2008). A New Model Selection Strategy In Artificial Neural Network, Applied Mathematics and Computation, 195, 591-597.

Egrioglu E., Aladag C.H., Yolcu U., Başaran A., \& Uslu V.R. (2009). A New Hybrid Approach Based on SARIMA and Partial High Order Bivariate Fuzzy Time Series Forecasting Model, Expert Systems with Applications, 36, 7424-7434.

Jordan, M.I. (1986). Serial order: A parallel distributed processing approach (Tech. Rep. No. 8604). San Diego: University of California, Institute for Cognitive Science.

Kennedy, J., Eberhart, R. (1995). Particle swarm optimization, In Proceedings of IEEE International Conference on Neural Networks, pages 1942-1948, Piscataway, NJ, USA, IEEE Press.

Khashei M., Bijari M. (2010). An artificial network (p, d, q) model for time series forecasting, Expert Systems with Applications, $37,479-489$.

Elman, J.L. (1990). Finding Structure in Time. Cognitive Science 14 (2), 179-211.

Yolcu U., Aladag C.H., \& Egrioglu E. (2013). A New Linear \& Nonlinear Artificial Neural Network Model for Time Series Forecasting, Decision support System, 54, 1340-1347.

Yadav R.N., Kalra P.K., \& John J. (2007). Time series prediction with single multiplicative neuron model, Applied Soft Computing, 7, 11571163.

Zhang, G., Patuwo, B.E., \& Hu, Y.M. (1998). Forecasting with artificial neural networks: The state of the art, International Journal of Forecasting, 14, 35-62.

Zhang G., Time series forecasting using a hybrid ARIMA and neural network model, Neurocomputing, 50 (2003) 159-175. 